\title{
On the bi-stable nature of turbulent premixed bluff-body stabilized flames at elevated pressure and near lean blow-off
}

\author{
Aaron W. Skiba ${ }^{\mathrm{a}, *}$, Thibault F. Guiberti ${ }^{\mathrm{b}}$, Wesley R. Boyette ${ }^{\mathrm{b}}$, \\ William L. Roberts ${ }^{\mathrm{b}}$, Epaminondas Mastorakos ${ }^{\mathrm{a}}$ \\ ${ }^{a}$ Engineering Department, University of Cambridge, Cambridge, CB2 1PZ, UK \\ ${ }^{b}$ King Abdullah University of Science and Technology (KAUST), CCRC, Thuwal \\ 23955-6900, Saudi Arabia
}

\begin{abstract}
This study considers turbulent premixed bluff-body stabilized flames at elevated pressures. Specifically, the lean blow-off (LBO) limit of such flames is determined for a range of bulk velocities $(5 \leq U \leq 50 \mathrm{~m} / \mathrm{s})$ and operating pressures up to 3 bar. Two key observations emerge from this stability assessment. The first is that considering elevated pressure leads to two stability regimes: one at atmospheric conditions and those with elevated pressure and $U \gtrsim 20 \mathrm{~m} / \mathrm{s}$ (regime-a), and another at elevated pressures with $U \lesssim 20 \mathrm{~m} / \mathrm{s}$ (regime-b). The second observation is that within these regimes, LBO limits are insensitive to pressure. Flames in regime-a (S-flames) are found to be more stable than those in regime-b (U-flames). Advanced image-based diagnostics were employed to understand reasons for this difference in stability. Flow field measurements indicate that U-flames are associated with an outer recirculation zone (ORZ) that formed as pressure increased but receded from
\end{abstract}

\footnotetext{
*Corresponding author: Aaron W. Skiba

Email address: skiba@umich.edu (Aaron W. Skiba)
} 
the burner as $U$ surpassed $\sim 20 \mathrm{~m} / \mathrm{s}$. PLIF images of $\mathrm{CH}_{2} \mathrm{O}$ and $\mathrm{OH}$ demonstrated that the ORZ interacts with U-flames such that their downstream regions are prevented from collapsing to the inner recirculation zone (IRZ). Furthermore, analysis of the OH-PLIF images indicate that U-flames possess larger turbulent consumption rates, helping them form large IRZs and rendering them more susceptible to influence from the ORZ. Results of highspeed $\mathrm{OH}^{*}$ imaging demonstrate that LBO events differ between U- and Sflames. Namely, while S-flames collapse to their IRZs during LBO, U-flames lift off from the burner, depleting their anchoring regions of reactions and hot products. Losing back-support in this region is what ultimately reduces the stability of U-flames. Finally, the reason U-flames lift off from the burner during LBO is elucidated by joint flow-flame measurements. Specifically, the anchoring regions of $\mathrm{U}$-flames reside in regions of large axial velocity, which likely stems from their enhanced burning rates.

Keywords:

Turbulent premixed combustion, Lean blow-off, Bluff-body stabilized, Elevated pressure, PLIF diagnostics

\section{Introduction}

Turbulent premixed bluff-body stabilized (TPBBS) flames are relevant to many practical propulsion and power production engines [1]. Bluff bodies are used in such engines as they promote flame stabilization. Namely, they produce recirculation zones $(\mathrm{RZs})$ that enhance flame stability by generating low-velocity regions and by providing the reactants with a constant ignition source. Yet, there are limits to which TPBBS can be stabilized. Accurately 
predicting these limits is crucial for developing efficient, low-emission engines.

For this reason, numerous experiments have studied phenomena associated with the lean blow-off (LBO) of TPBBS flames. Summaries of early works in this area are provided in Refs. $[1,2]$, yet since those articles, significant understanding has been gained through the use of advanced imageand laser-based diagnostics. For example, Chauduri et al. [3] applied planar laser-induced fluorescence (PLIF) imaging of $\mathrm{OH}$ simultaneously with particle image velocimitry (PIV) as well as $\mathrm{OH}$ chemiluminescence (hereinafter $\mathrm{OH}^{*}$ ) imaging to TPBBS flames close to LBO. Their efforts helped paint a phenomenological picture of the LBO process, namely that as LBO is approached the flame front recedes to the RZ and overlaps with the primary shear layer where it becomes subjected to high levels of tangential strain rate. They argued that this causes localized extinction events, which allow un-burned species to enter the RZ such that the amount of hot-products within it, and subsequently its ability to ignite incoming reactants, is diminished. Ultimately, this increases quenching and continually reduces the amount of products in the RZ until only small, isolated pockets remain. It was suggested that such pockets can linger in the RZ for tens of milliseconds and can partially re-ignite portions of the incoming reactants. Yet, at this point, stable combustion is never re-attained and the flame eventually extinguishes entirely. Confirmation of these latter claims was provided in Refs. [4, 5], where high-speed $(5 \mathrm{kHz}) \mathrm{OH}-\mathrm{PLIF}$ and $\mathrm{OH}^{*}$ imaging was employed to assess the transient nature of LBO.

These diagnostics are, however, incapable of reliably identifying local extinction events [6]. The product of simultaneously collected formaldehyde- 
$\left(\mathrm{CH}_{2} \mathrm{O}-\right)$ and $\mathrm{OH}-\mathrm{LIF}$ signals can distinguish such events since it provides a reasonable marker of the heat release rate (HRR) in premixed flames [6]. Kariuki et al. [7] exploited this fact to visualize peak HRR regions of TPBBS flames near LBO. Their findings confirm the occurrence of localized extinction events along the shear layer of such flames and that their frequency increases as LBO is approached. A recent set of experiments by Chowdhury et al. [8] corroborate these findings.

While the aforementioned studies have provided valuable insight, all of them were performed at atmospheric conditions. Yet, practical combustion devices typically operate at elevated pressures. Prior studies have considered the effects of elevated pressure on the LBO of TPBBS flames (see Refs. [1, 2, 9] and those therein); however, due to the unavailability of advance imaging diagnostics, such investigations were unable to correlate key structural features of such flames to their behavior near LBO. Hence, there is a need to increase understanding of the effects elevated pressure has on TPBBS flames near LBO. To address this need, this work considers axisymmetric TPBBS flames operated at elevated pressures and at conditions approaching LBO. A detailed assessment of these flames is facilitated by results from advanced diagnostics.

\section{Experimental details}

A diagram of the bluff-body burner employed here, which was replicated

from Refs. $[4,5,7]$, and an image of it in operation are provided in Fig. 1. To assess the effects of elevated pressure, this burner was operated within the High Pressure Combustion Duct (HPCD) at the Clean Combustion Re- 
search Center (CCRC) located at KAUST [10]. Such operation required shrouding the burner within an air coflow, the bulk velocity of which never exceeded $6 \%$ of that through the burner $(U)$ and was found to have no influence on flame stability. Only premixed methane-air flames were considered and their stability (i.e. LBO limit) was determined over a range of condtions with $5 \leq U \leq 50 \mathrm{~m} / \mathrm{s}$ and chamber pressures ranging from 1 to 3 bar. Here, pressure was varied by altering the flow of air through the shroud surrounding the co-flow and burner (see Fig. 1a). LBO limits were determined as in Ref. [4]. Namely, each flame was ignited at a stable equivalence ratio $(\phi)$ of 0.8 and then, keeping the air flow rate fixed, the fuel flow rate was incrementally decreased such that $\phi$ reduced by $\sim 3 \%$ every $\sim 30$ seconds until a flame was no longer visible. To elucidate observations from the stability assessment, detailed diagnostics were applied to seven flame conditions; the key parameters of which are listed in Table 1.

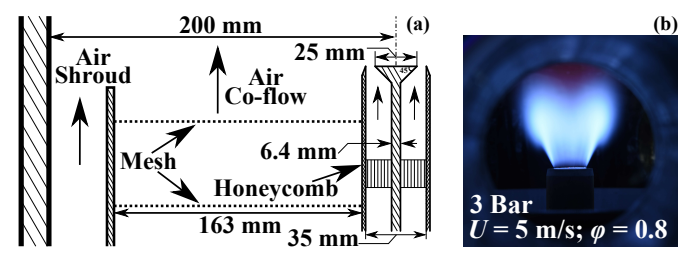

Figure 1: (a) Diagram of the burner within the HPCD. (b) Photo of the burner operating at 3 bar and with $U=5 \mathrm{~m} / \mathrm{s}$ and $\phi=0.8$.

Non- and reacting PIV measurements were made for all cases in Table 1 except those with $U=40 \mathrm{~m} / \mathrm{s}$. The turbulence parameters of those cases were estimated by assuming $u^{\prime}=0.17 U$ and that $\ell$ was similar to that in the lower velocity cases, where $u^{\prime}$ and $\ell$ represent the RMS of the velocity fluctuations and characteristic integral length scale [5], respectively. For a subset of these cases (i.e. the $1 \mathrm{~S}, 2 \mathrm{~S}$, and $2 \mathrm{U}$ cases), the PIV measurements were 


\begin{tabular}{cccccc}
\hline Case & $\phi$ & $u^{\prime} / S_{L}{ }^{a}$ & $\ell / \delta_{L}{ }^{b}$ & $R e_{T, 0}{ }^{c}$ & $S_{L}(\mathrm{~cm} / \mathrm{s})$ \\
\hline 1S-10.0 & 0.60 & 15.7 & 4.3 & 444 & 10.73 \\
1S-17.5 & 0.68 & 16.6 & 7.6 & 942 & 12.29 \\
1S-40.0* & 0.70 & 35.9 & 7.3 & 2000 & 18.95 \\
\hline 2U-10.0 & 0.73 & 11.1 & 7.6 & 637 & 16.49 \\
2S-17.5 & 0.66 & 26.2 & 7.9 & 1442 & 11.25 \\
\hline 3U-10.0 & 0.75 & 14.5 & 8.4 & 881 & 12.47 \\
3S-40.0* & 0.69 & 62.5 & 8.0 & 3500 & 10.88
\end{tabular}

Table 1: Details of the 7 cases investigated here. A case is referred to as $\alpha \beta-\gamma$, where $\alpha$ indicates the operating pressure (bar), $\beta$ denotes the flame type ( $\mathrm{S}$ and $\mathrm{U}$ for stable and unstable, respectively), and $\gamma$ represents the bulk velocity of that case $(\mathrm{m} / \mathrm{s}) \cdot u^{\prime} / S_{L}{ }^{a}$ is the turbulence level, where $S_{L}$ is the laminar flame speed computed via CHEMKIN with GRI-Mech 3.0. $\ell / \delta_{L}{ }^{b}$ is the characteristic length scale ratio, where $\delta_{L}$ is the computed laminar flame thickness. $R e_{T, 0}{ }^{c}$ is the turbulent Reynolds number defined as $u^{\prime} \ell / \nu$, where $\nu$ is the viscosity of the reactants. Turbulence parameters stem from non-reacting conditions at the locations marked by the green "Xs" in Fig. 5.

performed jointly with PLIF-imaging of OH. For the PIV measurements, the frequency-doubled output from a dual-pulse Nd:YAG laser (Litron Nano L 200-15 PIV) was formed into a 56 (tall) $\times 0.75$ (thick) $\mathrm{mm}^{2}$ laser sheet and directed over the mid-plane of the burner. The delay between the separate pulses was $28 \mu \mathrm{s}$ and $16 \mu \mathrm{s}$ for cases with $U=10$ and $17.5 \mathrm{~m} / \mathrm{s}$, respectively. Mie scattering from $0.5 \mu \mathrm{m} \mathrm{TiO}_{2}$ seeding particles were imaged by a CCD camera (LaVision ImagerIntense) with an array size of 1024 (tall) $\times 1376$ pixels $^{2}$ (wide) . The camera was fitted with a 60-mm f/2.8 Nikon lens and a $532 \pm 3 \mathrm{~nm}$ bandpass filter (Andover BP 532-3). The area imaged by the camera was 43 (tall) $\times 57$ (wide) $\mathrm{mm}^{2}$, yielding a projected pixel size of $41.5 \mu \mathrm{m}$. Commercial software (DaVis 8.4) was used to calculate vector fields from the particle images. The software was implemented with a multiple-pass, decreasing window, correlation-based computation scheme wherein the final 
window setting yielded an interrogation size and vector spacing of $500 \mu \mathrm{m}$ and $125 \mu \mathrm{m}$, respectively. Finally, post processing of the vector fields was performed in Matlab where an outlier detection scheme [11] was implemented without smoothing to remove spurious vectors.

The internal structure of a subset of the flames in Table 1 was visualized by employing simultaneous PLIF-imaging of $\mathrm{OH}$ and $\mathrm{CH}_{2} \mathrm{O}$. To image $\mathrm{CH}_{2} \mathrm{O}$-LIF signals, a 20 (tall) $\times 0.18$ (thick) $\mathrm{mm}^{2}$ laser sheet with pulse energies of $\sim 220 \mathrm{~mJ}$ was formed from the frequency-tripled output (near $355 \mathrm{~nm}$ ) of an Nd:YAG laser (Spectra Physics Quanta-Ray Pro 290). $\mathrm{CH}_{2} \mathrm{O}-\mathrm{LIF}$ signals resulting from the excitation of multiple transtions in the $4_{0}^{1}$ vibrational band of $\mathrm{CH}_{2} \mathrm{O}$ were imaged by an ICCD camera (PCO HSFC) with a 800 (tall) $\times 1280$ (wide) pixels $^{2}$ sensor, which was equipped with a $105-\mathrm{mm}$ f/2.8 lens (Nikon) and a multi-band filter (Semrock FF01-CH2O-50). An area of $56($ tall $) \times 90$ (wide) $\mathrm{mm}^{2}$ was imaged by this camera: thus its projected pixel size was $\sim 70 \mu \mathrm{m}$, while the image resolution was determined to be $\sim 260 \mu \mathrm{m}$. Excitation of $\mathrm{OH}$ was achieved by pumping the $\mathrm{Q}_{1}(6)$ transition in its $\mathrm{A}^{2} \Sigma^{+}-\mathrm{X}^{2} \Pi(1,0)$ band. This was performed by forming the frequencydoubled output of an Nd:YAG dye-pumped laser system (see Refs. [10] for details) to produce a 20 (tall) $\times 0.2$ (thick) $\mathrm{mm}^{2}$ laser sheet with a wavelength near $283 \mathrm{~nm}$. Fluorescence resulting from the excited $\mathrm{OH}$ was imaged by an ICCD camera (Princeton Instruments PI-MAX4) mounted opposite to the $\mathrm{CH}_{2} \mathrm{O}$ camera and outfitted with a 105-mm f/4.5 UV lens (Coastal optics) and a bandpass filter $(310 \pm 5 \mathrm{~nm}$; Asahi Spectra). This camera, with a $1024 \times 1024$ pixels $^{2}$ sensor, imaged an area of $52 \times 52 \mathrm{~mm}^{2}$ to yield a projected pixel size of $\sim 51 \mu \mathrm{m}$, though the resolution of each image was deter- 
mined to be $\sim 210 \mu \mathrm{m}$. The $355-\mathrm{nm}$ and $283-\mathrm{nm}$ laser sheets were carefully combined and overlapped in space but were delayed by $200 \mathrm{~ns}$ to avoid crosstalk. As mentioned, for a subset of the cases in Table 1, OH-PLIF imaging was performed jointly with the PIV measurements. Such OH-PLIF imaging was facilitated by the same system, the only differences being that a taller sheet was formed $(56 \mathrm{~mm})$, the camera was equipped with a $100-\mathrm{mm} \mathrm{f} / 2.8$ UV lens (Cerco), and the 283-nm laser pulse was delayed by $8 \mu$ s from the first PIV-laser pulse.

Processing of the PLIF images involved removal of signal from the camera offset, flame chemiluminescence, and un-rejected laser light. Corrections for vertical variations in the laser energy (derived from LIF signals wherein the target species were uniformly distributed over a large region) were then applied. Following those corrections, the PLIF images were subjected to a self-guided [12] and a median filter with $5 \times 5$ pixels $^{2}$ and $3 \times 3$ pixels $^{2}$ kernels, respectively, resulting in $\mathrm{SNR}$ of $\sim 15$ and $\sim 8$ for $\mathrm{OH}$ and $\mathrm{CH}_{2} \mathrm{O}$, respectively. Next, the OH-PLIF images were registered to the $\mathrm{CH}_{2} \mathrm{O}-\mathrm{PLIF}$ or vector field images $[13,14]$. Finally, as in prior studies of turbulent premixed flames (see, for example, Refs. [6-8, 13-16]), the processed OH- and $\mathrm{CH}_{2} \mathrm{O}-\mathrm{PLIF}$ images were multiplied on a pixel-by-pixel basis to yield "overlap-layers" [13], which roughly estimate regions of peak HRR [6].

\section{Results and discussion}

Figure 2 presents stability curves for the flames considered here. The horizontal and vertical error bars in Fig. 2 depict the uncertainty of the

pressure $( \pm 0.1$ bar $)$ within the HPCD and of the mass flow rates ( $\sim 3 \%$ of 
the set value), respectively. For comparison, the LBO limits determined in Ref. [5] (blue stars) are also provided in Fig. 2. Observing that their data points lie close to those obtained here under near-atmospheric conditions (black triangles) provides confidence in the results.

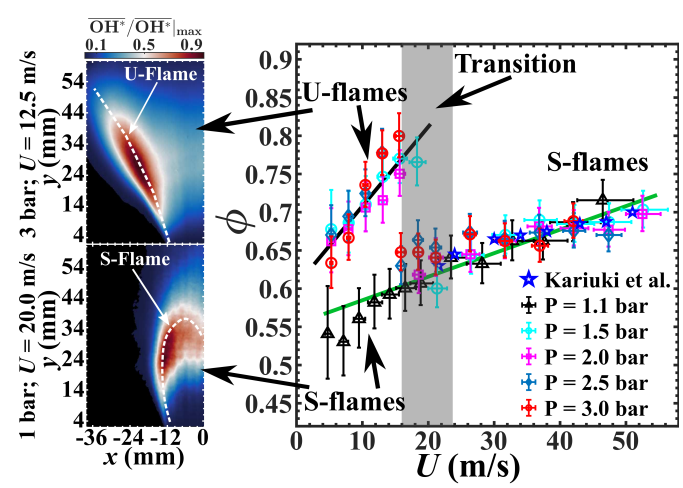

Figure 2: Stability curve. Error bars represent the accuracy of the flow rate through and pressure within the duct. Average $\mathrm{OH}^{*}$ images from a 3- (top) and 1.1-bar (bottom) flame are shown to the left.

Figure 2 indicates that for $U \gtrsim 20 \mathrm{~m} / \mathrm{s}$, the LBO limits of flames at elevated pressures closely coincide with those determined at- [5] and nearatmospheric (1.1 bar) conditions. Furthermore, they exhibit the same trend as in previous studies $[1,2,4]: \phi$ at LBO increases with $U$. Another observation from Fig. 2 is that when $U \lesssim 20 \mathrm{~m} / \mathrm{s}$, flames at elevated pressures blow off at larger $\phi$ than their near-atmospheric counterparts. Yet, as for the flames with $U \gtrsim 20 \mathrm{~m} / \mathrm{s}$, the LBO limits within this regime are independent of pressure. Also, at elevated pressures and $U \approx 20 \mathrm{~m} / \mathrm{s}$, some flames would initially appear like those with $U<20 \mathrm{~m} / \mathrm{s}$ but would transition to emulate, both in terms of appearance and stability, those with $U>20 \mathrm{~m} / \mathrm{s}$. Such transition was chaotic, but was less likely to occur when fuel-reduction rate increased. Overall, for the burner and conditions considered here, Fig. 2 
implies that elevating operating pressure yields two stability regimes, within which LBO limits are relatively insensitive to pressure variations.

To visualize differences between the flames within these separate regimes, high-speed $(5 \mathrm{kHz}) \mathrm{OH}^{*}$ imaging was employed. These images were collected with a high-speed camera (LaVision HighSpeedStar) equipped with a highspeed intensifier (LaVision IRO) and the same Cerco lens and bandpass filter as for the OH-PLIF measurements. The $\mathrm{OH}^{*}$ images were binned $2 \times 2$ after readout and were subjected to the same filtering process as the PLIF images. Average $\mathrm{OH}^{*}$ images from a 1.1- and 3.0-bar flame with $U=20 \mathrm{~m} / \mathrm{s}$ and $12.5 \mathrm{~m} / \mathrm{s}$ are shown in the bottom and top panels on the left side of Fig. 2, respectively. A clear difference between these separate flames is the overall shape they embody; the near-atmospheric flame is confined to its inner RZ (IRZ) whilst the 3-bar flame covers a much larger region with an open IRZ. OH* images from cases to the right of the gray region in Fig. 2 (see supplementary material) appear similar to that from the 1.1-bar case. This is true regardless of operating pressure, indicating that an important link exists between flame shape and stability, which has also been observed in swirl-stabilized flames [17-19].

Beyond differences in stability and appearance, the flames in the less and more stable regime (hereinafter referred to as U- and S-flames, respectively) experience contrasting events during LBO. This is evident from Fig. 3, which presents a sequence of $\mathrm{OH}^{*}$ images from the data used to generate the average images in Fig. 2. It is apparent from Fig. 2a that as LBO was approached, the S-flame receded to the IRZ where pockets of $\mathrm{OH}^{*}$ signal remained close to the bluff body before vanishing entirely. This is consistent with Refs. [3-5]; 
yet, it is quite different from what is seen in Fig. 3b, which shows a similar sequence for a U-flame. Instead of retreating to the IRZ, prior to LBO this flame lifted off from the burner leaving the key flame-anchoring region void of hot products and reactions.

To support these observations, the area-integrated $\mathrm{OH}^{*}$ signal (normalized by its average value prior to LBO; $I_{O H^{*}}$ ), and the lift-off height (i.e. the vertical distance from the bluff-body to the closest point of non-zero $\mathrm{OH}^{*}$ signal) are plotted against time in Fig. 3c. The lift-off behavior of the Uflame (dashed-red line) is apparent from this plot. As this flame approached LBO it retreated from and propagated back towards the burner several times. This plot also indicates that final blow-off of the U-flame is associated with $\mathrm{OH}^{*}$ pockets being carried far (>1 bluff-body diameter) downstream of the burner. In contrast, before $\mathrm{LBO}, \mathrm{OH}^{*}$ pockets in the S-flame (dashed-blue line) remain within a few millimeters of the bluff body. Additionally, $I_{O H^{*}}$ curves in Fig. 3c imply that the level of reaction fluctuates considerably more about its mean for the S- (solid-blue line) than for the U-flame (solidred line). This is consistent with the images in Fig. 3a and is indicative of the cyclic extinction/re-ignition phenomena observed previously [3-5]. The lower extent of $I_{O H^{*}}$ fluctuations in the U-flame align with the observation from Fig. 3c that relatively large regions of strong $\mathrm{OH}^{*}$ signal tend to persist even when the flame is lifted. Suggesting that this flame experienced less large-scale extinction/re-ignition events, which may result from a lack of reactions/hot-products within its primary anchoring region.

Figure 4 displays instantaneous and average $\mathrm{CH}_{2} \mathrm{O}$ - and $\mathrm{OH}-\mathrm{PLIF}$ images from flames 1S-10.0 (top) and 3U-10.0 (bottom) in Table 1. The average 


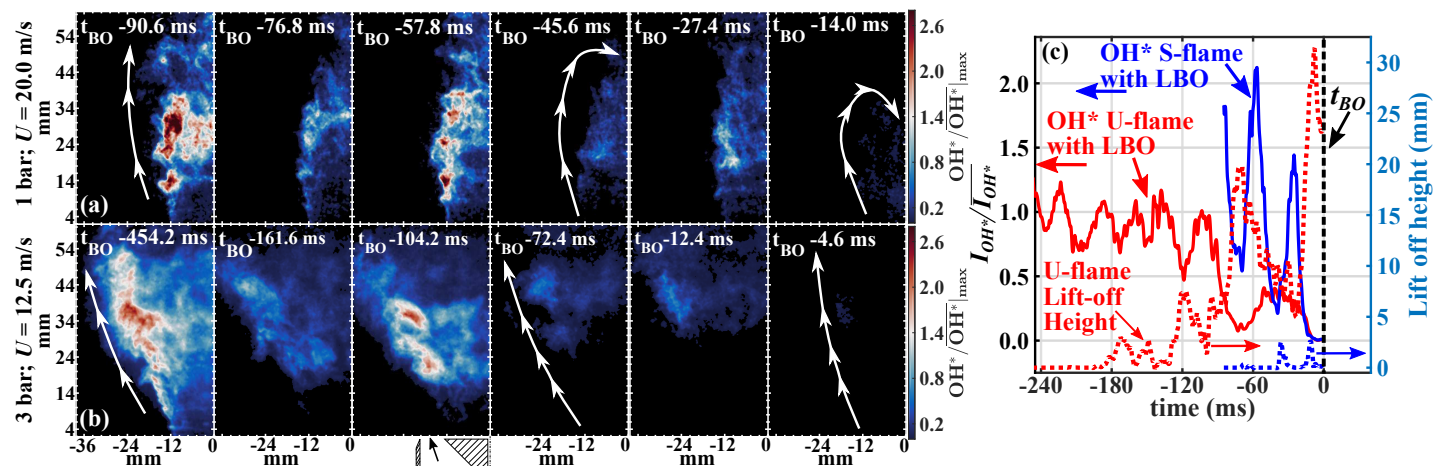

Figure 3: $\mathrm{OH}^{*}$ sequences and quantities derived from them. Videos of these sequences are provided in the supplementary material

images in Fig. 4, as well as the other statistical quantities shown below, stem from an ensemble of 1000 instantaneous frames. The PLIF images in Figs. 4ad clearly highlight the structural difference between the S- and U-flames. In S-flames, hot products $(\mathrm{OH})$ are confined to the IRZ and un-burned species $\left(\mathrm{CH}_{2} \mathrm{O}\right)$ begin to make their way there as the axial distance $(y)$ increases. Conversely, the IRZ of the U-flame is broad, filled with hot products, and widens with $y$. Moreover, no $\mathrm{CH}_{2} \mathrm{O}$ is found within the IRZ of this flame. Consequently, its IRZ is void of primary reactions, which is supported by the average overlap-layer image in Fig. 4d. This feature is unique to the U-flame as the S-flame considered here (see Fig. 4c), like those in Ref. [7], possesses reaction layers within its IRZs whilst near LBO.

Another difference between the U- and S-flames in Fig. 4 is the broad region of $\mathrm{CH}_{2} \mathrm{O}$ the $\mathrm{U}$-flame has within and beyond its outer shear layer. The average axial velocity fields $\left(\overline{V_{y}}\right)$ in the rightmost column of Fig. 4 indicate that such $\mathrm{CH}_{2} \mathrm{O}$ results from an outer $\mathrm{RZ}$ (ORZ) that forms in the U-flame. From Fig. 5, which presents $\overline{V_{y}}$-fields from non-reacting conditions, it is apparent that this ORZ also exists in the absence of combustion. Fur- 


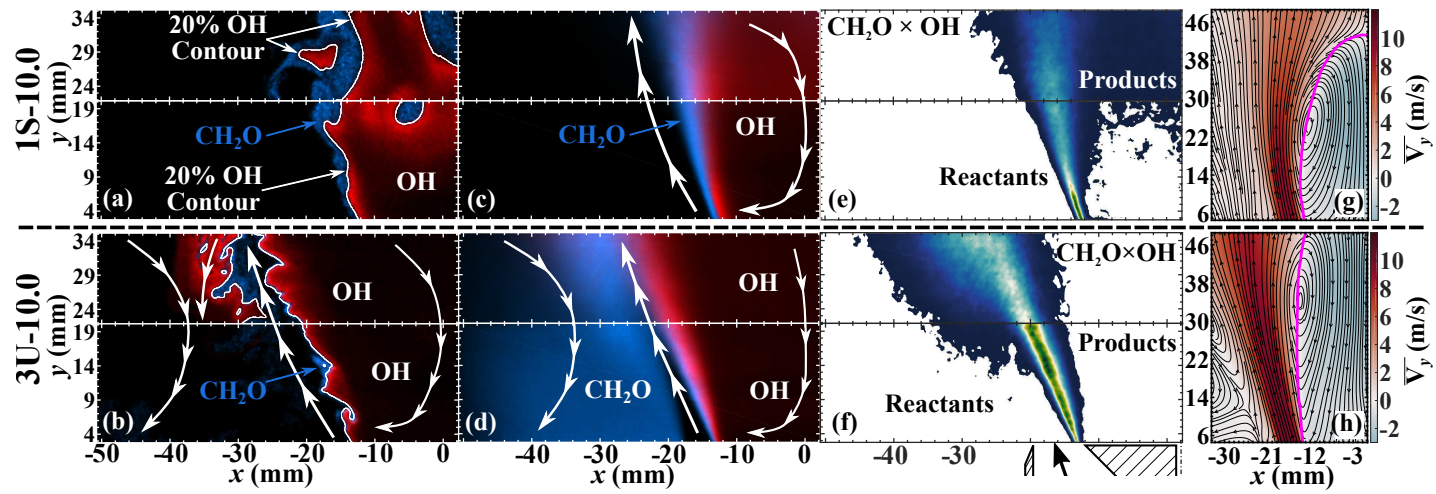

Figure 4: From left to right: Instantaneous then average $\mathrm{CH}_{2} \mathrm{O}$ - (blue) and OH-PLIF (red) images, average overlap-layer images, and average $V_{y}$ fields (reacting) from cases 1S-10.0 (top) and 3U-10.0 (bottom).

ther inspection of Fig. 5 reveals that an ORZ is characteristic of conditions associated with U-flames: only cases at elevated pressure with $U \lesssim 20 \mathrm{~m} / \mathrm{s}$ exhibit an ORZ. Additionally, the ORZ of the 1.5-bar cases in Fig. 5 is observed to move away from the burner as $U$ increases. This occurrence was also observed in the other elevated pressure cases (not shown). Accordingly, the observations from Figs. 4 and 5 suggest that U-flames are a consequence of changes to the aerodynamics of the system, which depend on both chamber pressure and the momentum through the burner. Specifically, as indicated by the instantaneous images in Fig. 4b (and confirmed by joint OH-PLIF/PIV images in the supplementary material), the ORZ interacts with the downstream region (i.e. $y>25 \mathrm{~mm}$ ) of U-flames, causing them to wrap over the reactant jet. This interaction is one likely reason U-flames burn with a much wider IRZ than S-flames, particularly in downstream regions.

Comparison of the $\overline{V_{y}}$-fields in Figs. 4 and 5 demonstrates that combustion enlarges the IRZ, which stems from volumetric expansion across the flame [1]. U-flames clearly exhibit larger IRZs than S-flames, rendering their 


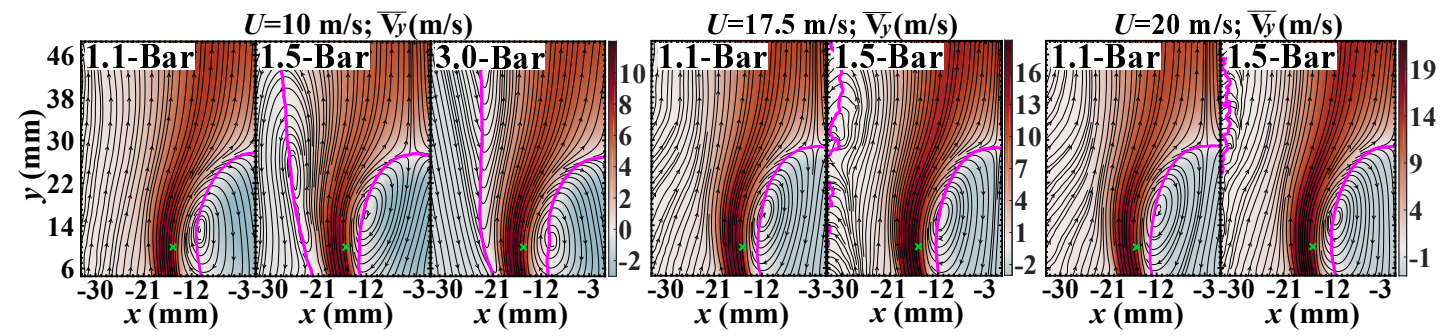

Figure 5: Non-reacting $\overline{V_{y}}$-fields. Magenta lines mark $V_{y}=0$. Green "Xs" mark where parameters in Table 1 were extracted.

downstream regions more susceptible to influence from the ORZ. While the ORZ likely facilitates widening of downstream regions of U-flames, their anchoring regions are also wider than those in S-flames. This is highlighted by the average progress variable $(\bar{C})$ maps in Fig. 6a, which represent the average of OH-PLIF images binarized based on their $20 \%$ contours. These maps demonstrate that the U-flame burns at a more acute angle with respect to the horizontal $(\beta)$ than its S-flame counterpart, which is corroborated by the plot of $\beta$ (derived from $\bar{C}=0.5$ contours) vs. $y$ in Fig. $6 \mathrm{~b}$. This observation suggests that for the same $U$, a U-flame likely possesses a larger average turbulent flame speed $\left(\overline{S_{T}}\right)$ than its associated S-flame. To assess this, an approach similar to that in Ref. [20] was used to determine local turbulent consumption speeds $\left(S_{T, L C}\right)$ of flames 1S-10.0, 3U-10.0, 1S-40.0, and 3S-40.0 in Table 1. Namely, values of $S_{T, L C} / S_{L}$ were computed within the flame anchoring region by integrating two-dimensional (2-D) average flame surface density (FSD; $\bar{\Sigma}$ ) measurements in the horizontal direction and accounting for the angle between the local average flame-front normal and the horizontal (see Eq. A2 in Ref. [20]). Here, a "sliding-box" method [14] was applied to instantaneous flame fronts, defined as the $20 \%$ OH-PLIF contours (i.e. white lines in Figs. 4a and 4b), to produce 2-D $\Sigma$-fields that were averaged to obtain 
$\bar{\Sigma}$.

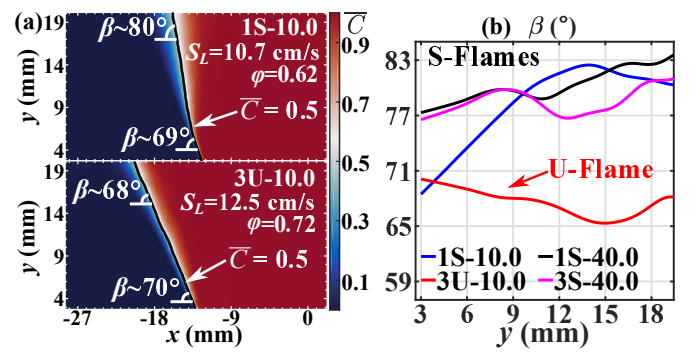

Figure 6: (a) $\bar{C}$-maps of flames 1S-10.0 (top) and 3U-10.0 (bottom). (b) Angle of $\bar{C}=0.5$ countours $(\beta)$ vs. $y$.

Results of this analysis are displayed in Figs. 7a and 7b, which plot $S_{T, L C} / S_{L}$ and $S_{T, L C} / U$ as a function of $y$, respectively. It is apparent from Fig. 7 that flame 3U-10.0 (red lines) possess larger values of $S_{T, L C} / S_{L}$ and $S_{T, L C} / U$ than its corresponding S-flame (1S-10.0; blue lines). The reason for this enhanced propagation rate can be understood from panels (c) and (d) of Fig. 7, which present flame-front curvature $(\kappa)$ and $\bar{\Sigma}$ results, respectively. It is clear from Fig. 7c that the U-flame possess a broader $\kappa$-PDF (where $\kappa$ is derived based on the same formulation and definition as in Ref. [7]) than its corresponding S-flame. This implies that elevating pressure results in a higher degree of wrinkling, which is in line with Refs. [21-23]. More flame surface wrinkling indicates that the U-flames possess more flame surface overall, which is evident from Fig. 7d where the U-flame exhibits the largest $\bar{\Sigma}$ values. Based on Damköhler's first hypothesis, more flame surface area corresponds to a greater consumption rate [20, 24, 25]. Indeed, Fig. 7a indicates that at low bulk velocities, increasing the pressure from 1.1 to 3.0 bar leads to an average increase of $\sim 20 \%$ in $S_{T, L C} / S_{L}$, which is also consistent with prior results [23]. 
The enhanced consumption rates of U-flames may also contribute to their characteristic shape. Namely, while the ORZ appears to prevent the downstream region of U-flames from retreating to their IRZs, their enhanced $S_{T, L C} / S_{L}$ values likely reduced the $\beta$ they burn with, resulting in a wide IRZ. Additionally, by burning with a smaller $\beta$, the downstream regions of U-flames are forced to reside in close proximity to the ORZ, thus increasing the potential for flame-ORZ interactions in this region. The fact U-flames do not persist for all $U$ at elevated pressure may also be linked to $S_{T}$; namely, its sublinear growth with increased $u^{\prime} / S_{L}[24,25]$. Here, $u^{\prime}$ increases as $\sim 0.17 U$ (see Table 1), yet beyond a particular turbulence level, $S_{T}$ increases less rapidly with $u^{\prime}[24,25]$. Thus, it is plausible that this "bending" attribute of $S_{T}$ results in flames at elevated pressure and $U \gtrsim 20 \mathrm{~m} / \mathrm{s}$ to burn with a larger $\beta$, thereby reducing the influence of the ORZ and allowing them to retreat to their IRZs during LBO. This is supported by the black and magenta lines in Fig. 6b, which plot $\beta$ from flames 1S-40.0, and 3S-40.0, respectively.
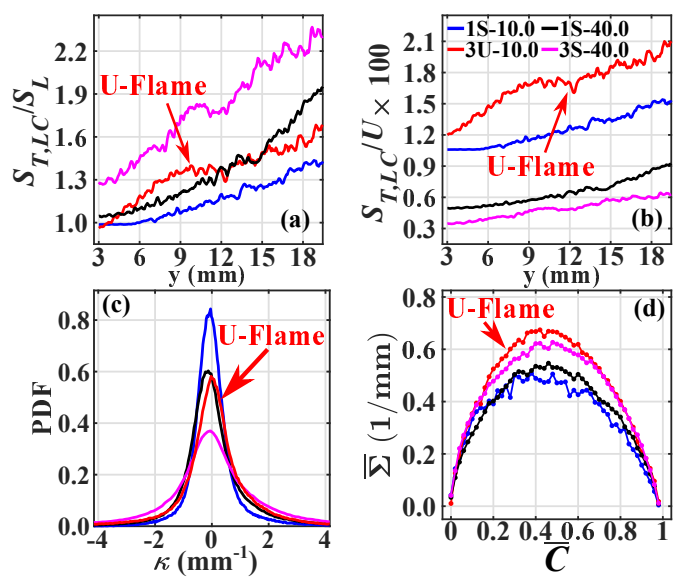

Figure 7: Flame surface characteristics and turbulent flame speeds. 
While the previous assessment sheds light on why U-flames form, it does not explain their reduced stability. In fact, Damköhler number scaling principles $[1,2]$ suggest that flames with larger IRZs, like U-flames, should be more stable than those with smaller IRZs. Results from joint flow-flame measurements can, however, shed light on this matter. For instance, consider Figs. 8a-d, which display $\overline{V_{y}}$-fields from flames 1S-10.0, 2U-10.0, 1S-17.5, and 2S-17.5. The cyan lines superimposed on these maps mark the $0.2,0.5$, and $0.8 \bar{C}$-contours. By comparing Fig. 8b to Figs. 8a, 8c, and 8d, it is apparent that more of the U-flame sits within regions of large $\overline{V_{y}}$ than the S-flames. This is particularly true within the primary anchoring region (i.e. $y<20 \mathrm{~mm}$ ). This observation is corroborated by Fig. 8e, which plots PDFs of $V_{y}$ conditioned on the instantaneous flame fronts normalized by their respective $U$. Unlike the PDFs for the S-flames, which are broadly distributed between $U / 2$ and $U$, that from flame $2 \mathrm{U}-10.0$ displays a predominant peak near its $U$ value. The fact that $\mathrm{U}$-flames tend to be subjected to larger $V_{y}$ values than their corresponding S-flames likely explains why they tend to lift off from the burner during LBO, which from Fig. 3 appears to be closely connected to their reduced degree of stability.

Results and notions presented above are now combined to yield a phenomenological explanation for the occurrence and reduced stability of Uflames. Namely, in this study increasing pressure results in the formation of an ORZ that interacts with the downstream regions of U-flames, preventing this region from collapsing to the IRZ. This interaction is seemingly enhanced by the increased consumption rates of U-flames. That is, as in prior studies [21-23], for a given $U$, elevating pressure increases flame surface area, re- 
sulting in larger $S_{T, L C} / S_{L}$ values. The effect of increased $S_{T, L C} / S_{L}$ is likely two-fold: 1) it allows the anchoring region of U-flames to burn with a smaller $\beta$; and 2) it positions the downstream region of the flame closer to the ORZ. These effects likely compound to inhibit U-flames from retreating to their IRZs during LBO. Also, the enhanced consumption rates of U-flames allows them to reside within regions of large $V_{y}$. Concequently, U-flames lift off from the burner, leaving their anchoring region void of reactions and hot products to reignite them. The lack of reignition events, which are common in S-flames during LBO [3-5], ultimately inhibits re-stabilization of U-flames, leading to total blow-off. Finally, the reason U-flames do not persist for all $U$ at elevated pressure is also linked to the ORZ and $S_{T}$. Namely, as $U$ increases, the ORZ is forced away from the flames. Additionally, the "bending" attribute of $S_{T}$ $[24,25]$ with increased turbulence level likely contributes to the observation that flames at larger $U$ burn with a larger $\beta$, thus decreasing the potential for flame-ORZ interactions.
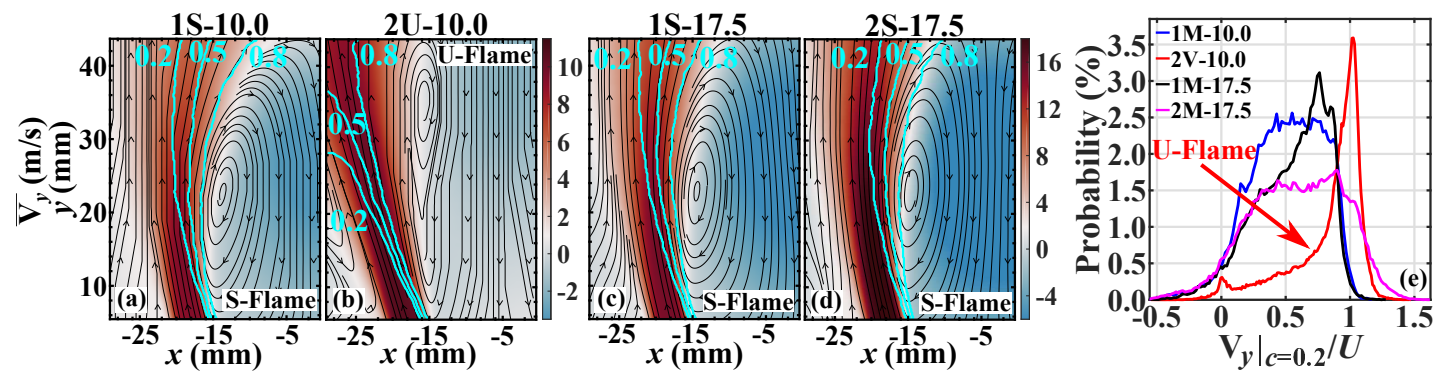

Figure 8: Average axial velocity profiles with $\bar{C}$-contours overlaid and PDFs of axial velocity conditioned on the instantaneous flame fronts. 


\section{Conclusions}

This study considered the effects of elevated pressure (up to 3 bar) on turbulent premixed bluff-body stabilized flames near lean blow-off (LBO). A principal finding was that elevating pressure leads to the formation of two stability regimes with: a) atmospheric conditions or elevated pressures with bulk velocities $(U)$ above $\sim 20 \mathrm{~m} / \mathrm{s}$, and b) elevated pressures and $U \lesssim 20 \mathrm{~m} / \mathrm{s}$. Yet, within these separate regimes the LBO limits were relatively insensitive to pressure. Flow field measurements indicated that regime-b, which is less stable than regime-a, results from an outer recirculation zone (ORZ) that formed as pressure increased but was forced away from the burner when $U$ exceeded $\sim 20 \mathrm{~m} / \mathrm{s}$. PLIF imaging of $\mathrm{CH}_{2} \mathrm{O}$ and $\mathrm{OH}$ demonstrated that the ORZ interacts with the downstream region $(y>20 \mathrm{~mm})$ of flames in regimeb (U-flames). Further analysis of the OH-PLIF images indicated that Uflames possess larger local turbulent consumption speeds $\left(S_{T, L C}\right)$ than their counterparts in regime-a (S-flames). Combined, these phenomena cause Uflames to burn with much larger inner recirculation zones (IRZs) than Sflames. Furthermore, high-speed $\mathrm{OH}^{*}$ imaging indicated that U-flames blowoff in a different manner than S-flames. Namely, S-flames recede to their IRZs as LBO is approached, whereas U-flames lifted off from the burner, leaving their anchoring regions void of primary reactions and hot products. The loss of back-support in this region is the ultimate reason U-flames are less stable than S-flames. Finally, joint PIV and OH-PLIF measurements indicated that the behavior of U-flames during LBO is a consequence of their anchoring regions being situated in areas of large axial velocity $\left(V_{y}\right)$. The reason U-flames reside in areas of large $V_{y}$ is likely a result of their larger 
$S_{T, L C}$ values.

\section{Acknowledgments}

Research reported in this publication was supported by funding from the King Abdullah University of Science and Technology (KAUST).

\section{References}

[1] S. J. Shanbhogue, S. Husain, T. Lieuwen, Lean blowoff of bluff body stabilized flames : Scaling and dynamics, Prog. Energy Combust. Sci. 35 (2009) 98-120.

[2] K. Radhakrishnan, J. B. Heywood, R. J. Tabaczynski, Premixed turbulent flame blowoff velocity correlation gased on coherent structures in turbulent flows, Combust. Flame 42 (1981) 19-33.

[3] S. Chaudhuri, S. Kostka, M. W. Renfro, B. M. Cetegen, Blowoff dynamics of bluff body stabilized turbulent premixed flames, Combust. Flame 157 (2010) 790-802.

[4] J. R. Dawson, R. L. Gordon, J. Kariuki, E. Mastorakos, A. R. Masri, M. Juddoo, Visualization of blow-off events in bluff-body stabilized turbulent premixed flames, Proc. Combust. Inst. 33 (2011) 1559-1566.

[5] J. Kariuki, J. R. Dawson, E. Mastorakos, Measurements in turbulent premixed bluff body flames close to blow-off, Combust. Flame 159 (2012) 2589-2607. 
[6] P. H. Paul, H. N. Najm, Planar laser-induced fluorescence imaging of flame heat release rate, Proc. Combust. Inst. 27 (3) (1998) 43-50.

[7] J. Kariuki, A. Dowlut, R. Yuan, R. Balachandran, E. Mastorakos, Heat release imaging in turbulent premixed methane-air flames close to blowoff, Proc. Combust. Inst. 35 (2) (2015) 1443-1450.

[8] B. R. Chowdhury, B. M. Cetegen, Effects of fuel properties and free stream turbulence on characteristics of bluff-body stabilized flames, Combust. Flame 194 (2018) 206-222.

[9] J. P. Longwell, J. E. Chenevey, W. W. Clark, E. E. Frost, Flame stabilization by baffles in a high velocity gas stream, Symp. (Int.) Combust. 3 (1949) 40-43.

[10] W. R. Boyette, T. F. Guiberti, G. Magnotti, W. L. Roberts, Structure of turbulent nonpremixed syngas flames at high pressure, Proc. Combust. Inst. 37 (2019) 2207-2214.

[11] D. Garcia, A fast all-in-one method for automated post-processing of PIV data, Exp. Fluids 50 (2011) 1247-1259.

[12] K. He, J. Sun, X. Tang, Guided image filtering, IEEE Trans. Pattern Anal. Mach. Intell. 35 (6) (2013) 1397-1409.

[13] A. W. Skiba, T. M. Wabel, C. D. Carter, S. D. Hammack, J. E. Temme, J. F. Driscoll, Premixed flames subjected to extreme levels of turbulence part I: Flame structure and a new measured regime diagram, Combust. Flame 189 (2018) 407-432. 
[14] A. W. Skiba, T. M. Wabel, C. D. Carter, S. D. Hammack, J. E. Temme, T. Lee, J. F. Driscoll, Reaction layer visualization: A comparison of two PLIF techniques and advantages of kHz-imaging, Proc. Combust. Inst. 36 (2017) 4593-4601.

[15] M. J. Dunn, A. R. Masri, R. W. Bilger, R. S. Barlow, Finite rate chemistry effects in highly sheared turbulent premixed flames, Flow, Turbul. Combust. 85 (3-4) (2010) 621-648.

[16] B. Zhou, C. Brackmann, Z. Wang, Z. Li, M. Richter, M. Aldén, X.-S. Bai, Thin reaction zone and distributed reaction zone regimes in turbulent premixed methane/air flames: Scalar distributions and correlations, Combust. Flame 175 (2017) 220-236.

[17] S. Hermeth, G. Staffelbach, L. Y. M. Gicquel, V. Anisimov, C. Cirigliano, T. Poinsot, Bistable swirled flames and influence on flame transfer functions, Combust. Flame 161 (2014) 184-196.

[18] Q. An, W. Y. Kwong, B. D. Geraedts, A. M. Steinberg, Coupled dynamics of lift-off and precessing vortex core formation in swirl flames 168 (2016) 228-239.

[19] I. Boxx, C. M. Arndt, C. D. Carter, W. Meier, High-speed laser diagnostics for the study of flame dynamics in a lean premixed gas turbine model combustor, Exp. Fluids 52 (2012) 555-567.

[20] S. Kheirkhah, Ö. L. Gülder, Consumption speed and burning velocity in counter-gradient and gradient diffusion regimes of turbulent premixed combustion, Combust. Flame 162 (4) (2015) 1422-1439. 
[21] A. Soika, F. Dinkelacker, A. Leipertz, Pressure influence on the flame front curvature of turbulent premixed flames : comparison between experiment and theory, Combust. Flame 132 (2003) 451-462.

[22] T. Lachaux, F. Halter, C. Chauveau, I. Go, I. G. Shepherd, Flame front analysis of high-pressure turbulent lean premixed methane air flames, Proc. Combust. Inst. 30 (2005) 819-826.

[23] J. Wang, S. Yu, M. Zhang, W. Jin, Z. Huang, S. Chen, H. Kobayashi, Burning velocity and statistical flame front structure of turbulent premixed flames at high pressure up to 1.0 MPa, Exp. Therm. Fluid Sci. 68 (2015) 196-204.

[24] J. F. Driscoll, J. H. Chen, A. W. Skiba, C. D. Carter, E. R. Hawkes, H. Wang, Premixed flames subjected to extreme turbulence: Some questions and recent answers, Prog. Energy Combust. Sci. 76 (2020).

[25] G. Nivarti, S. Cant, Direct Numerical Simulation of the bending effect in turbulent premixed flames, Proc. Combust. Inst. 36 (2017) 1903-1910. 